\title{
Editorial
}

\section{Starting treatment with angiotensin converting enzyme inhibitors in heart failure: how, where, and how much?}

The benefits of angiotensin converting enzyme inhibitors (ACE inhibitors) in symptomatic heart failure are now well established. The CONSENSUS, ${ }^{1}$ SOLVD treatment arm, ${ }^{2}$ and VHeft $\mathrm{II}^{3}$ studies all showed that enalapril improved outcome and exercise tolerance in patients with symptomatic chronic heart failure and low ejection fractions. Treatment with an ACE inhibitor should now be considered standard in patients with symptomatic heart failure despite treatment with diuretics or digitalis therapy or both. What is now of interest to the clinician is the evidence that lies behind this recommendation.

\section{Clinical questions}

What are the contraindications to ACE inhibitors; which is the best agent and are there significant differences between them; which dose should we use; should all patients be started on treatment in hospital, and how should this best be done? Unfortunately, specific randomised controlled trials are unlikely to be performed to answer these questions. It could be argued that the National Health Service should direct research and development funds to hospitals or units that are prepared and able to undertake research to answer these clinical questions. Such research could easily pay for itself in the longer term by avoiding widespread prescribing based on supposition rather than fact. In the likely continued absence of specific trial data we must attempt to answer these clinically important questions by a review of the facts that are known.

\section{Evidence}

MacFadyen and colleagues (pages 293-7) ask whether treatment with ACE inhibitors can be safely started as a low dose intravenous infusion in patients with stable heart failure ${ }^{4}$ They propose that this might be a way of safely titrating the dose of ACE inhibitor for individual patients. They have tested their proposal in a clinically relevant group-namely those likely to be at increased risk of first dose hypotension-elderly patients with heart failure taking substantial doses of diuretic. This and an earlier companion paper $^{5}$ are also rare in being randomised placebo controlled trials of treatment regimens rather than of individual drugs. Intravenous initiation of ACE inhibitors is not common and this trial is too small to determine whether this approach causes less serious side effects than conventional oral initiation. Nor does it prove that the reduction in blood pressure with an intravenous pro-drug will predict the hypotension seen after the first oral dose. MacFadyen et al do, however, show interesting differences both between the active ACE inhibitors and against the oral form of the pro-drug. ${ }^{5}$ Though the effects of plasma concentrations on haemodynamic function are interesting, it is doubtful whether the data presented justify the conclusions that starting treatment with intravenous pro-drug improves the safety of starting oral treatment. No adverse events were record- ed but few subjects were studied and there was no control group given conventional oral treatment that had a greater rate of complications. So we cannot assume that the regimen proposed was necessarily safer. We were not told of the haemodynamic effects of the eventual oral therapy, whether the results of the intravenous phase were used to select the eventual oral dose or whether a group given titrated intravenous pre-dosing had a smaller fall in blood pressure in response to an equivalent oral dose than a group that was not "pre-dosed."

MacFadyen et al conclude that "a formal trial of this (intravenous) approach (to ACE inhibitor initiation) in a further high risk population is justified." The routine use of this approach in clinical practice without the results of such a trial is, however, not justified. As MacFayden et al concluded in their earlier paper" "patterns of test dosing with captopril have been suggested but the individual relevance of this strategy to subsequent responses to higher doses of the same or different agents has not been documented." The same can be said of intravenous initiation of treatment with ACE inhibitors.

\section{Implications}

In large trials where most patients (over $98 \%$ of 2569 patients) were started on oral ACE inhibitor as outpatients important first dose adverse reactions were rare. ${ }^{2}$ Clearly it is not feasible or necessary to recommend that all patients starting ACE inhibitor treatment do so as inpatients. Recommendations such as the routine admission of all patients requiring ACE inhibitor initiation may even be partly responsible for the very low rate of ACE inhibitor treatment in the United Kingdom. If there is a long wait to see a specialist and an even longer wait to be admitted, patients and their doctors may never get around to starting treatment.

It is unlikely that we will see major mortality trials to compare similar ACE inhibitors in heart failure or asymptomatic left ventricular dysfunction. If the analysis of all available randomised placebo controlled and active agent controlled trials for a new ACE inhibitor does not show significant adverse effects on mortality when a test agent is compared with established agents, and if benefits in terms of symptoms, exercise performance, or other physiological end points thought to be useful are proven, then these new agents should receive licences for the same indications as the established ACE inhibitors. Some clinicians will use only those agents that have been shown to reduce mortality: others will favour newer agents with supposed and highly promoted advantages. We cannot expect consensus in this choice. If the choice of antihypertensive agent were restricted to those known to reduce mortality our pharmacopoeia would be much reduced. In heart failure any agent which is adequately shown to improve symptoms and patient performance should receive approval provided it can be shown that its effect on outcome is no worse than that of placebo or other established ACE inhibitors. 
In asymptomatic left ventricular dysfunction, however, the evidence for benefit should be stronger, requiring both prevention of disability and either a proven reduction in mortality or a similar effect as an agent of the same drug class that is known to reduce mortality. The new agent should in the latter case be "on probation" with careful post-marketing surveillance of its effects on mortality. Captopril has been shown to reduce mortality when started in carefully selected subjects but only after a considerable length of treatment. ${ }^{6}$ Early intravenous enalapril followed by oral therapy did not produce the same benefit. ${ }^{7}$ The reasons for this difference are not clear because there were too many other differences between the two trials. These included the selection of patients, the timing, the route of administration, the duration of action of the agent, and the duration of follow up of the patients. We should be wary, however, of over-emphasising the positive trial and under-quoting the larger negative trial. In patients with acute myocardial infarction the titrated dose achieved with the intravenous approach avoids rapid uncontrollable reductions in blood pressure, but as yet there is no evidence that we should even attempt to initiate the ACE inhibitor therapy soon after infarction. Preventing remodelling and fibrosis may be beneficial later but may be harmful when early repair is taking place. In the CONSENSUS II trial intravenous dosing of enalaprilat ( $1 \mathrm{mg}$ over 2 hours) did not prevent a tendency to increased mortality and an increased frequency of hypotensive reactions early after infarction. ${ }^{7}$

We will probably never know whether all ACE inhibitors have similar effects on patients with symptomatic heart failure. The benefits shown for enalapril in three heart failure mortality trials (two against placebo) probably apply to all ACE inhibitors that have been shown to improve symptoms and performance. In fact in some head-to-head comparisons captopril has been suggested to have advantages over the agent more extensively studied in mortality trials, enalapril. ${ }^{8}$ Agents will inevitably differ in ancillary properties and smaller mech- anistic studies may demonstrate differences between ACE inhibitors in duration of action; tissue specificity; and effects on renal function, muscle blood flow, ventilatory control, exercise performance, and haemodynamic function. It is right that these factors should be taken into account in the choice of agent for individual patients.

Without more direct funding of clinical research we may never learn the answers to some of the most basic of questions about treatment. Research sponsored by the pharmaceutical industry will not necessarily address the questions that the National Health Service wants answered-such as, when is it best to start treatment and what is the dose-response of the beneficial effects of ACE inhibitors in heart failure.

National Heart and Lung Institute,

ANDREW J S COATS

Dovehouse Street,

London SW3 6LY

1 The CONSENSUS Trial Study Group.Effects of enalapril on mortality in severe congestive heart failure: results of the Cooperative North
Scandinavian Enalapril Survival Study. $N$ Engl $f$ Med 1987;316: 1429-35.

2 The SOLVD Investigators. Effect of enalapril on survival in patients with reduced left ventricular ejection fractions and congestive heart failure. $N$ Engl f Med 1991;325:293-302.

3 Cohn JN,. Johnson G, Ziesche S, et al. A comparison of enalapril with hydralazine-isosorbide dinitrate in the treatment of chronic congestive heart failure. $N$ Engl f Med 1991;325:303-10.

$4 \mathrm{MacF}$ adyen RJ, Lees KR, Reid JL. A double blind controlled study of low dose intravenous peridoprilat or enalaprilat infusion in elderly patients with heart failure. Br Heart $\mathcal{F}$ 1993;69:293-7.

5 MacFadyen RJ, Lees KR, Reid JL. Differences in first dose response to angiotensin converting enzyme inhibition in congestive heart failure: a placebo controlled study. Br Heart $\mathcal{f}$ 1991;66:206-11.

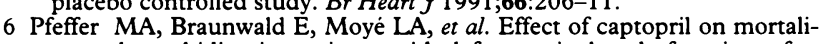
ty and morbidity in patients with left ventricular dysfunction after ty and morbidity in patients with left ventricular dysfunction after
myocardial infarction. Results of the Survival and Ventricular myocardial infarction. Results of the Survival
Enlargement Trial. $N$ Engl $\mathcal{F}$ Med 1992;327:669-77.

7 Swedberg K, Held P, Kjekshus J, et al. Effects of the early administration of enalapril on mortality in patients with acute myocardial infarction, results of the Cooperative New Scandinavian Enalapril Survival Study II (CONSENSUS II). $N$ Engl $\mathcal{F}$ Med 1992;327:678-84.

8 Packer M, Lee WH, Yushak M, Medina N. Comparison of captopril and enalapril in patients with severe chronic heart failure. $N$ Engl $\mathcal{F}$ Med 1986;315:847-53. 